\title{
SPHERICAL STRAIN STATE OF INCOMPRESSIBLE ELASTIC BODIES
}

\author{
István Ecsedi AND AtTila BAKsA \\ University of Miskolc \\ Institute of Applied Mechanics, Hungary \\ istvan.ecsedi@uni-miskolc.hu, attila.baksa@uni-miskolc.hu
}

[Received: February 29, 2016 Accepted: May 5, 2016]

\begin{abstract}
This paper is concerned with the statics of an isotropic, linearly elastic, homogeneous incompressible body. The body considered is bounded by two spherical surfaces and two conical surfaces. The centre of the spherical surfaces and the apex of conical surfaces are the same point. The concept of the spherical strain state is introduced and the concerning boundary value problem of elastic equilibrium is formulated in terms of a displacement potential and the mean stress. Two examples illustrate the applications of the derived equations. The analytical solutions we have obtained are compared with FEM solutions computed by the Abaqus finite element software.
\end{abstract}

Mathematical Subject Classification: 70B10, 70B15

Keywords: Spherical strain state, incompressible, linearly elastic

\section{INTRODUCTION}

As is well known the displacement based finite element solutions for incompressible isotropic elastic bodies have some difficulties such as the Poisson ratio locking [1, 2. Poisson (volumetric) locking is a serious problem which cannot be avoided by refining the mesh. All the standard fully integrated finite elements will lock in the incompressible limit, and some elements show very poor performance if the Poisson ratio $\nu$ is approximately 0.5 . The source of the error of the finite element solution when the Poisson ratio $\nu$ is close to 0.5 is that the finite element interpolation functions are unable to properly approximate a volume preserving strain field [1. Therefore it is of great importance to find exact analytical solutions for some statical problems of incompressible elastic bodies which then can be compared with the finite element solutions. The aim of this paper is to give a new class of the exact benchmark solutions for incompressible elastic bodies.

The present paper is concerned with a statical boundary value problem of an isotropic, homogeneous, incompressible elastic body. The body considered is bounded by two spherical surfaces $\partial V_{1}, \partial V_{2}$ and two conical surfaces $\partial V_{3}, \partial V_{4}$. The centre of the spherical surfaces $\partial V_{1}, \partial V_{2}$ and the apex of the conical surfaces $\partial V_{3}, \partial V_{4}$ are the 
same point $O$ as shown in Figure 1. A spherical polar coordinate system $\operatorname{Or} \varphi \vartheta$ is introduced with an origo $O$ (Figure 1). The body shown in Figure 1 is said to be in the Spherical Strain State (SSS) if its displacement field has the form

$$
u_{r}=0, \quad u_{\vartheta}=r U_{\vartheta}(\vartheta, \varphi), \quad u_{\varphi}=r U_{\varphi}(\vartheta, \varphi),
$$

where $u_{r}, u_{\vartheta}$ and $u_{\varphi}$ are the components of the displacement vector $\boldsymbol{u}=u_{r} \boldsymbol{e}_{r}+$ $u_{\varphi} \boldsymbol{e}_{\varphi}+u_{\vartheta} \boldsymbol{e}_{\vartheta}$ and $\boldsymbol{e}_{r}, \boldsymbol{e}_{\varphi}$ and $\boldsymbol{e}_{\vartheta}$ are the unit vectors of the spherical coordinate system $\operatorname{Or} \varphi \vartheta$. Application of the strain-displacement relationships of the linearised theory of elasticity leads to the next results for the strains in the spherical coordinate system $\operatorname{Or} \varphi \vartheta$ 4,5.

$$
\begin{gathered}
\varepsilon_{r}=0, \quad \varepsilon_{\vartheta}(\vartheta, \varphi)=\frac{\partial U_{\vartheta}}{\partial \vartheta}, \quad \varepsilon_{\varphi}(\vartheta, \varphi)=\frac{1}{\sin \vartheta} \frac{\partial U_{\varphi}}{\partial \varphi}+U_{\vartheta} \cot \vartheta \\
\gamma_{r \varphi}=\gamma_{r \vartheta}=0, \quad \gamma_{\varphi \vartheta}(\vartheta, \varphi)=\frac{1}{\sin \vartheta} \frac{\partial U_{\vartheta}}{\partial \varphi}+\frac{\partial U_{\varphi}}{\partial \vartheta}-U_{\varphi} \cot \vartheta
\end{gathered}
$$

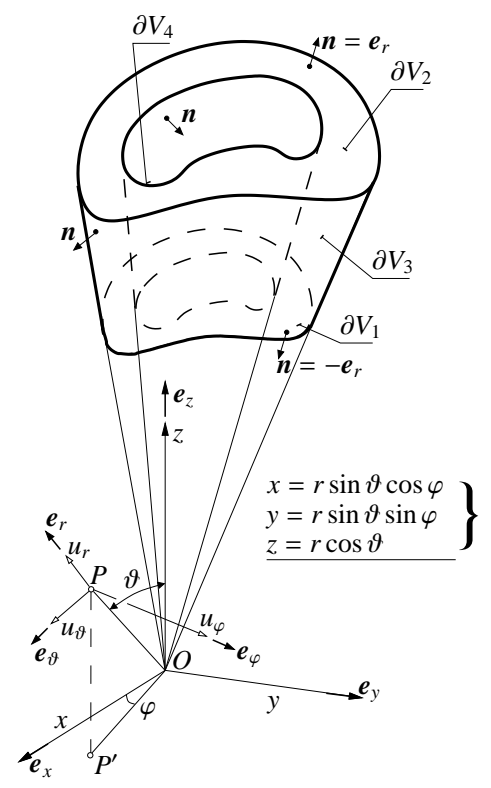

Figure 1. Space domain bounded by spherical and conical surfaces

The material of the body considered is homogeneous, isotropic, incompressible and linearly elastic. According to Hooke's law for $\nu=0.5$ we can write 3

$$
\begin{aligned}
\sigma_{r} & =2 G \varepsilon_{r}+\sigma_{0}, \\
\sigma_{\varphi} & =2 G \varepsilon_{\varphi}+\sigma_{0}, \\
\sigma_{\vartheta} & =2 G \varepsilon_{\vartheta}+\sigma_{0},
\end{aligned}
$$




$$
\begin{gathered}
\tau_{r \varphi}=G \gamma_{r \varphi}, \quad \tau_{r \vartheta}=G \gamma_{r \vartheta}, \\
\tau_{\varphi \vartheta}=G \gamma_{\varphi \vartheta},
\end{gathered}
$$

where $\sigma_{r}, \sigma_{\varphi}$ and $\sigma_{\vartheta}$ are the normal stresses, $\tau_{r \varphi}, \tau_{r \vartheta}$ and $\tau_{\varphi \vartheta}$ are the shearing stresses, $G$ is the shear modulus of elasticity and $\sigma_{0}$ is the mean stress defined by the following equation

$$
\sigma_{0}=\frac{1}{3}\left(\sigma_{r}+\sigma_{\varphi}+\sigma_{\vartheta}\right) .
$$

For incompressible elastic body in SSS we have 3

$$
\operatorname{div} \boldsymbol{u}=\varepsilon_{r}+\varepsilon_{\varphi}+\varepsilon_{\vartheta}=\frac{\partial U_{\vartheta}}{\partial \vartheta}+\frac{1}{\sin \vartheta} \frac{\partial U_{\varphi}}{\partial \varphi}+U_{\vartheta} \cot \vartheta=0 .
$$

\section{BOUNDARY VALUE PROBLEM}

The following prescriptions define the boundary value problem of the elastic equilibrium we shall consider,

- There is no body forces in $V$, where $V$ is the space domain occupied by body shown in Figure 1.

- The boundary conditions are given by the following equations:

$$
\begin{aligned}
u_{r}=0 \quad \text { and } \tau_{r \varphi}=\tau_{r \vartheta}=0 \quad \text { on } \partial V_{1} \cup \partial V_{2}, \\
\boldsymbol{p}_{3}=\boldsymbol{T} \cdot \boldsymbol{n}, \quad \boldsymbol{p}_{3}=\widetilde{\boldsymbol{p}}_{3} \quad \text { on } \quad \partial V_{3} \quad \text { or } \quad \boldsymbol{u}=\widetilde{\boldsymbol{u}}_{3} \quad \text { on } \partial V_{3}, \\
\boldsymbol{p}_{4}=\boldsymbol{T} \cdot \boldsymbol{n}, \quad \boldsymbol{p}_{4}=\widetilde{\boldsymbol{p}}_{4} \quad \text { on } \quad \partial V_{4} \quad \text { or } \quad \boldsymbol{u}=\widetilde{\boldsymbol{u}}_{4} \quad \text { on } \partial V_{4},
\end{aligned}
$$

The applied traction vector $\widetilde{\boldsymbol{p}}_{i}(i=3,4)$ and the prescribed surface displacement $\widetilde{\boldsymbol{u}}_{i}$ on the boundary surface segment $\partial V_{i}(i=3,4)$ have the forms

$$
\begin{gathered}
\widetilde{\boldsymbol{p}}_{i}=\widetilde{p}_{i \vartheta}(\vartheta, \varphi) \boldsymbol{e}_{\vartheta}+\widetilde{p}_{i \varphi}(\vartheta, \varphi) \boldsymbol{e}_{\varphi} \quad(i=3,4), \\
\widetilde{\boldsymbol{u}}_{i}=r\left(\widetilde{U}_{i \vartheta}(\vartheta, \varphi) \boldsymbol{e}_{\vartheta}+\widetilde{U}_{i \varphi}(\vartheta, \varphi) \boldsymbol{e}_{\varphi}\right) \quad(i=3,4) .
\end{gathered}
$$

We remark that the radial coordinate $r$ is constant on the boundary parts $\partial V_{i}$ $(i=1,2)$, that is, $r=r_{i}$ where $r_{i}$ is a constant. Observe that the traction vectors $\widetilde{\boldsymbol{p}}_{3}$ and $\widetilde{\boldsymbol{p}}_{4}$ cannot be prescribed arbitrarily, they should statisfy an equation. Later on we shall derived this equation from the condition of global mechanical equilibrium.

In the present case the local equilibrium condition can be given in the following form [4.5]:

$$
\boldsymbol{T} \cdot \nabla=\mathbf{0} \text { in } V
$$

where $\nabla$ is the gradient (del) operator. By applying the Gauss divergence theorem we get from equation 2.6 that

$$
\int_{V} \boldsymbol{T} \cdot \nabla \mathrm{d} V=\int_{\partial V} \boldsymbol{T} \cdot \boldsymbol{n} \mathrm{d} A=\int_{\partial V_{1}} \boldsymbol{T} \cdot \boldsymbol{n} \mathrm{d} A+\int_{\partial V_{2}} \boldsymbol{T} \cdot \boldsymbol{n} \mathrm{d} A+\int_{\partial V_{3}} \boldsymbol{T} \cdot \boldsymbol{n} \mathrm{d} A+\int_{\partial V_{4}} \boldsymbol{T} \cdot \boldsymbol{n} \mathrm{d} A=\mathbf{0} .
$$


Here $\boldsymbol{n}$ is the outer unit normal vector on the boundary surface $\partial V=\bigcup_{i=1}^{4} \partial V_{i}$ and $\mathrm{d} A$
is the area element:

$$
\begin{aligned}
& \mathrm{d} A=r_{i}^{2} \sin \vartheta \mathrm{d} \vartheta \mathrm{d} \varphi=r_{i}^{2} \mathrm{~d} a \quad \text { and } \quad \boldsymbol{n}=(-1)^{i} \boldsymbol{e}_{r} \quad \text { on } \quad \partial V_{i}(i=1,2), \\
& \mathrm{d} A=r \mathrm{~d} r \mathrm{~d} s_{i} \quad \text { and } \quad \boldsymbol{n}=n_{\vartheta}(\vartheta, \varphi) \boldsymbol{e}_{\vartheta}+n_{\varphi}(\vartheta, \varphi) \boldsymbol{e}_{\varphi} \quad \text { on } \quad \partial V_{i}(i=3,4) .
\end{aligned}
$$

In equations 2.8 $\mathrm{a}, \mathrm{b}) \mathrm{d} a$ is the area element on a unit sphere and $\mathrm{d} s_{i}(i=3,4)$ is the arc element on the intersection line of the conical surface $\partial V_{i}(i=3,4)$ and the unit sphere $(r=1)$. It follows from equation (2.7) that the force equilibrium equation for the whole body assumes the following form

$$
\begin{aligned}
\int_{\partial V} \boldsymbol{T} \cdot \boldsymbol{n} \mathrm{d} A= & -\int_{\partial V_{1}} \sigma_{r} \boldsymbol{e}_{r} \mathrm{~d} A+\int_{\partial V_{2}} \sigma_{r} \boldsymbol{e}_{r} \mathrm{~d} A+\int_{\partial V_{3}} \boldsymbol{p}_{3} \mathrm{~d} A+\int_{\partial V_{4}} \boldsymbol{p}_{4} \mathrm{~d} A= \\
& \left(r_{2}^{2}-r_{1}^{2}\right) \int_{a} \sigma_{r} \boldsymbol{e}_{r} \mathrm{~d} a+\frac{r_{2}^{2}-r_{1}^{2}}{2}\left(\int_{\partial a_{3}} \boldsymbol{p}_{3} \mathrm{~d} s_{3}+\int_{\partial a_{4}} \boldsymbol{p}_{4} \mathrm{~d} s_{4}\right)=\mathbf{0},
\end{aligned}
$$

where the hollow two-dimensional domain $a$ on the unit sphere $0<\vartheta<\pi$ is bounded by the intersection line of the conical surfaces $\partial V_{3}, \partial V_{4}$ and the unit sphere. It is clear from Figure 2 that the boundary curve of $a$ is $\partial a=\partial a_{3} \cup \partial a_{4}$. A detailed form of equation 2.9 is given by

$$
2 \int_{a} \sigma_{r}(\vartheta, \varphi) \boldsymbol{e}_{r} \sin \vartheta \mathrm{d} \vartheta \mathrm{d} \varphi+\int_{\partial a_{3}} \boldsymbol{p}_{3}\left(s_{3}\right) \mathrm{d} s_{3}+\int_{\partial a_{4}} \boldsymbol{p}_{4}\left(s_{4}\right) \mathrm{d} s_{4}=\mathbf{0}
$$

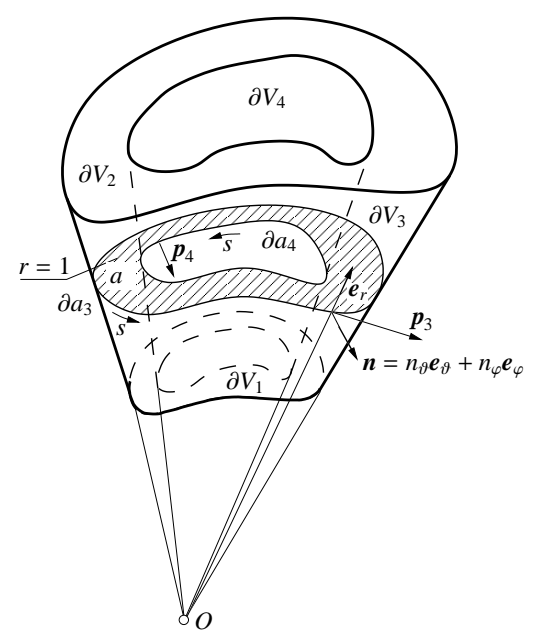

Figure 2. Definition of domain $a$ on the unit ball.

Equation 2.10 shows how the surface forces $\widetilde{\boldsymbol{p}}_{3}, \widetilde{\boldsymbol{p}}_{4}$ are related to the stresses on the boundary surface $\partial V_{1} \cup \partial V_{2}$. We would like to emphasise that equation 2.9 does not provide any constraint for the applied surface forces given by relation 2.4. 
In the absence of body forces the local equilibrium condition

$$
\boldsymbol{r} \times \boldsymbol{T} \cdot \nabla=\mathbf{0} \quad\left(\boldsymbol{r}=r \boldsymbol{e}_{r}\right)
$$

can be transformed into a global one in the same way as we did for equation 2.6 . We obtain

$$
\begin{array}{r}
\int_{V} \boldsymbol{r} \times \boldsymbol{T} \cdot \nabla \mathrm{d} V=\int_{\partial V} \boldsymbol{r} \times \boldsymbol{T} \cdot \boldsymbol{n} \mathrm{d} A-\int_{V}\left(\boldsymbol{e}_{x} \times \boldsymbol{T} \cdot \boldsymbol{e}_{x}+\boldsymbol{e}_{y} \times \boldsymbol{T} \cdot \boldsymbol{e}_{y}+\boldsymbol{e}_{z} \times \boldsymbol{T} \cdot \boldsymbol{e}_{z}\right) \mathrm{d} V \\
=\int_{\partial V} \boldsymbol{r} \times \boldsymbol{T} \cdot \boldsymbol{n} \mathrm{d} A=\mathbf{0}, \quad(2.12)
\end{array}
$$

where we have taken into account that [4,5]:

$$
\boldsymbol{e}_{x} \times \boldsymbol{T} \cdot \boldsymbol{e}_{x}+\boldsymbol{e}_{y} \times \boldsymbol{T} \cdot \boldsymbol{e}_{y}+\boldsymbol{e}_{z} \times \boldsymbol{T} \cdot \boldsymbol{e}_{z}=\mathbf{0},
$$

Here $\boldsymbol{e}_{x}, \boldsymbol{e}_{y}$ and $\boldsymbol{e}_{z}$ are the unit vectors of the Cartesian coordinate system Oxyz shown in Figure 1 .

It follows from equations 2.11 and 2.12 that

$$
\begin{gathered}
\int_{\partial V} \boldsymbol{r} \times \boldsymbol{T} \cdot \boldsymbol{n} \mathrm{d} A=\int_{\partial V_{1}} \boldsymbol{r} \times \boldsymbol{T} \cdot \boldsymbol{n} \mathrm{d} A+\int_{\partial V_{2}} \boldsymbol{r} \times \boldsymbol{T} \cdot \boldsymbol{n} \mathrm{d} A+\int_{\partial V_{3}} \boldsymbol{r} \times \boldsymbol{p}_{3} \mathrm{~d} A+\int_{\partial V_{4}} \boldsymbol{r} \times \boldsymbol{p}_{4} \mathrm{~d} A= \\
-r_{1} \int_{\partial V_{1}} \boldsymbol{e}_{r} \times \sigma_{r} \boldsymbol{e}_{r} \mathrm{~d} A+r_{2} \int_{\partial V_{2}} \boldsymbol{e}_{r} \times \sigma_{r} \boldsymbol{e}_{r} \mathrm{~d} A+\int_{r_{1}}^{r_{2}} r^{2}\left(\int_{\partial a_{3}} \boldsymbol{e}_{r} \times \boldsymbol{p}_{3} \mathrm{~d} s_{3}\right) \mathrm{d} r+ \\
\int_{r_{1}}^{r_{2}} r^{2}\left(\int_{\partial a_{4}} \boldsymbol{e}_{r} \times \boldsymbol{p}_{4} \mathrm{~d} s_{4}\right) \mathrm{d} r=\mathbf{0 .} \quad(2.14)
\end{gathered}
$$

The moment equilibrium equation 2.14 we have derived can be rewritten into a more compact form

$$
\int_{\partial a_{3}} \boldsymbol{e}_{r} \times \boldsymbol{p}_{3} \mathrm{~d} s_{3}+\int_{\partial a_{4}} \boldsymbol{e}_{r} \times \boldsymbol{p}_{4} \mathrm{~d} s_{4}=\mathbf{0} .
$$

This equation is the constraining equation the applied surface forces $\widetilde{\boldsymbol{p}}_{3}$ and $\widetilde{\boldsymbol{p}}_{4}$ should meet. Consequently, for the boundary value problem we have defined in this section the constraint equation 2.15 we have established is a solvability condition.

For the displacement boundary conditions of the form

$$
\boldsymbol{u}=\widetilde{\boldsymbol{u}}_{3}=r \widetilde{\boldsymbol{U}}_{3} \quad \text { on } \quad \partial V_{3} \quad \text { and } \quad \boldsymbol{u}=\widetilde{\boldsymbol{u}}_{4}=r \widetilde{\boldsymbol{U}}_{4} \quad \text { on } \quad \partial V_{4}
$$

we can rewrite the solvability condition 2.15 by utilising the incompressiblity condition 1.10 :

$$
\int_{\partial a_{3}} \tilde{\boldsymbol{U}}_{3} \cdot \boldsymbol{n} \mathrm{d} s_{3}+\int_{\partial a_{4}} \tilde{\boldsymbol{U}}_{4} \cdot \boldsymbol{n} \mathrm{d} s_{4}=\mathbf{0} .
$$


3. Formulation of elastic Equilibrium in terms of Displacement POTENTIAL AND MEAN STRESS

In our formulation the unknown fields are $U_{\vartheta}=U_{\vartheta}(\vartheta, \varphi), U_{\varphi}=U_{\varphi}(\vartheta, \varphi)$ and $\sigma_{0}=\sigma_{0}(\vartheta, \varphi)$. Let $F=F(\vartheta, \varphi)$ be a displacement potential in the terms of which

$$
U_{\vartheta}=\frac{1}{\sin \vartheta} \frac{\partial F}{\partial \varphi}, \quad U_{\varphi}=-\frac{\partial F}{\partial \vartheta} .
$$

are the two displacement components. It follows from equations $1.2,(1.3)$ and 3.1 that the strains are

$$
\begin{gathered}
\varepsilon_{\vartheta}=\frac{\partial}{\partial \vartheta}\left(\frac{1}{\sin \vartheta} \frac{\partial F}{\partial \varphi}\right), \\
\varepsilon_{\varphi}=-\frac{\partial}{\partial \vartheta}\left(\frac{1}{\sin \vartheta} \frac{\partial F}{\partial \varphi}\right)
\end{gathered}
$$

and

$$
\gamma_{\varphi \vartheta}=\frac{1}{\sin ^{2} \vartheta} \frac{\partial^{2} F}{\partial \varphi^{2}}-\sin \vartheta \frac{\partial}{\partial \vartheta}\left(\frac{1}{\sin \vartheta} \frac{\partial F}{\partial \vartheta}\right) .
$$

For a sufficiently smooth otherwise arbitrary displacement potential $F=F(\vartheta, \varphi)$ the condition of incompressiblity

$$
\varepsilon_{r}+\varepsilon_{\vartheta}=0
$$

is identically satisfied. Upon substitution of equations 3.2 , (3.3) and (3.4) into Hooke's law 1.4 1.9 we get the stresses in terms of the displacment potential:

$$
\begin{gathered}
\sigma_{\vartheta}=2 G \frac{\partial}{\partial \vartheta}\left(\frac{1}{\sin \vartheta} \frac{\partial F}{\partial \varphi}\right)+\sigma_{0}, \\
\sigma_{\varphi}=-2 G \frac{\partial}{\partial \vartheta}\left(\frac{1}{\sin \vartheta} \frac{\partial F}{\partial \varphi}\right)+\sigma_{0}, \\
\sigma_{0}=\sigma_{r}=\frac{1}{2}\left(\sigma_{\vartheta}+\sigma_{\varphi}\right), \\
\tau_{\varphi \vartheta}=G\left[\frac{1}{\sin ^{2} \vartheta} \frac{\partial^{2} F}{\partial \varphi^{2}}-\sin \vartheta \frac{\partial}{\partial \vartheta}\left(\frac{1}{\sin \vartheta} \frac{\partial F}{\partial \vartheta}\right)\right], \\
\tau_{r \varphi}=\tau_{r \vartheta}=0 .
\end{gathered}
$$

It is well known that the equilibrium equations $(2.6)$ in spherical polar coordinate system are as follows 3,4$]$ :

$$
\begin{aligned}
\frac{\partial \sigma_{r}}{\partial r}+ & \frac{1}{r} \frac{\partial \tau_{\vartheta r}}{\partial \vartheta}+\frac{1}{r \sin \vartheta} \frac{\partial \tau_{\varphi r}}{\partial \varphi}+\frac{1}{r}\left(2 \sigma_{r}-\sigma_{\vartheta}-\sigma_{\varphi}+\tau_{\vartheta r} \cot \vartheta\right)=0 \\
\frac{\tau_{\vartheta r}}{\partial r}+\frac{1}{r} \frac{\partial \sigma_{\vartheta}}{\partial \vartheta}+\frac{1}{r \sin \vartheta} \frac{\partial \tau_{\varphi \vartheta}}{\partial \varphi}+\frac{1}{r}\left[\left(\sigma_{\vartheta}-\sigma_{\varphi}\right) \cot \vartheta+3 \tau_{r \vartheta}\right] & =0 \\
\frac{\partial \tau_{r \varphi}}{\partial r}+\frac{1}{r} \frac{\partial \tau_{\varphi \vartheta}}{\partial \vartheta}+\frac{1}{r \sin \vartheta} \frac{\partial \sigma_{\varphi}}{\partial \varphi}+\frac{1}{r}\left(2 \tau_{\varphi \vartheta} \cot \vartheta+3 \tau_{r \varphi}\right) & =0
\end{aligned}
$$


For SSS problems these equations assume the form

$$
\begin{gathered}
\frac{\partial \sigma_{\vartheta}}{\partial \vartheta}+\frac{1}{\sin \vartheta} \frac{\partial \tau_{\varphi \vartheta}}{\partial \varphi}+\left(\sigma_{\vartheta}-\sigma_{\varphi}\right) \cot \vartheta=0, \\
\frac{\partial \tau_{\varphi \vartheta}}{\partial \vartheta}+\frac{1}{\sin \vartheta} \frac{\partial \sigma_{\varphi}}{\partial \varphi}+2 \tau_{\varphi \vartheta} \cot \vartheta=0,
\end{gathered}
$$

since $\sigma_{\vartheta}=\sigma_{\vartheta}(\vartheta, \varphi), \sigma_{\varphi}=\sigma_{\varphi}(\vartheta, \varphi)$ and $\sigma_{r}=\sigma_{0} \equiv \sigma_{0}(\vartheta, \varphi)$.

A combination of equations (3.6), (3.7) and (3.8) with equations (3.14) and (3.15) yields a coupled system of differential equations for $F=F(\vartheta, \varphi)$ and $\sigma_{0}=\sigma_{0}(\vartheta, \varphi)$ :

$$
\begin{gathered}
\frac{1}{G} \frac{\partial \sigma_{0}}{\partial \vartheta}+L_{\vartheta}[F]=0, \\
\frac{1}{G} \frac{\partial \sigma_{0}}{\partial \varphi}+L_{\varphi}[F]=0,
\end{gathered}
$$

where

$$
\begin{aligned}
& L_{\vartheta}[F]=2 \frac{\partial^{2}}{\partial \vartheta^{2}}\left(\frac{1}{\sin \vartheta} \frac{\partial F}{\partial \varphi}\right)+\frac{1}{\sin ^{3} \vartheta} \frac{\partial^{3} F}{\partial \varphi^{3}}-\frac{\partial}{\partial \vartheta}\left(\frac{1}{\sin \vartheta} \frac{\partial^{2} F}{\partial \vartheta \partial \varphi}\right)+4 \cot \vartheta \frac{\partial}{\partial \vartheta}\left(\frac{1}{\sin \vartheta} \frac{\partial F}{\partial \varphi}\right), \\
& L_{\varphi}[F]= \frac{\partial}{\partial \vartheta}\left(\frac{1}{\sin ^{2} \vartheta} \frac{\partial^{2} F}{\partial \varphi^{2}}\right)-\frac{\partial}{\partial \vartheta}\left[\sin \vartheta \frac{\partial}{\partial \vartheta}\left(\frac{1}{\sin \vartheta} \frac{\partial F}{\partial \vartheta}\right)\right] \\
&-\frac{2}{\sin \vartheta} \frac{\partial}{\partial \vartheta}\left(\frac{1}{\sin \vartheta} \frac{\partial^{2} F}{\partial \varphi^{2}}\right)+2 \frac{\cot \vartheta}{\sin ^{2} \vartheta} \frac{\partial^{2} F}{\partial \varphi^{2}}-2 \cos \vartheta \frac{\partial}{\partial \vartheta}\left(\frac{1}{\sin \vartheta} \frac{\partial F}{\partial \vartheta}\right) .
\end{aligned}
$$

For a simply connected region $a$ fulfillment of the Schwarz theorem

$$
\frac{\partial}{\partial \vartheta}\left(\frac{\partial \sigma_{0}}{\partial \varphi}\right)=\frac{\partial}{\partial \varphi}\left(\frac{\partial \sigma_{0}}{\partial \vartheta}\right)
$$

assures that the function $\sigma_{0}=\sigma_{0}(\vartheta, \varphi)$ is determined with the accuracy of an arbitrary constant of integration $\bar{\sigma}_{0}=\sigma_{0}\left(\vartheta_{0}, \varphi_{0}\right)$ which is the value of the function $\sigma_{0}=\sigma_{0}(\vartheta, \varphi)$ at the point $P_{0}$.

For a double connected region $a(0<\vartheta<\pi)$ - this is our case - fulfillment of condition (3.20) is necessary but not sufficient. The additional condition to be satisfied is of the form

$$
\int_{\partial a_{4}} \frac{\partial \sigma_{0}}{\partial \vartheta} \mathrm{d} \vartheta+\int_{\partial a_{4}} \frac{\partial \sigma_{0}}{\partial \varphi} \mathrm{d} \varphi=0 .
$$

A combination of equations (3.16), (3.17) and (3.20), (3.21) results in the following two equations:

$$
\begin{gathered}
\frac{\partial L_{\vartheta}[F]}{\partial \varphi}-\frac{\partial L_{\varphi}[F]}{\partial \vartheta}=0 \quad(\vartheta, \varphi) \in a, \\
\int_{\partial a_{4}} L_{\vartheta}[F] \mathrm{d} \vartheta+\int_{\partial a_{4}} L_{\varphi}[F] \mathrm{d} \varphi=0 .
\end{gathered}
$$


With the knowledge of the displacement potential $F=F(\vartheta, \varphi)$ the mean stress is

$$
\sigma_{0}(\vartheta, \varphi)=\sigma_{0}\left(\vartheta_{0}, \varphi_{0}\right)-G\left(\int_{\widehat{P_{0} P}} L_{\vartheta}[F] \mathrm{d} \vartheta+\int_{\widehat{P_{0} P}} L_{\varphi}[F] \mathrm{d} \varphi\right),
$$

where $\widehat{P_{0} P}$ is an arbitrary curve in $a$ with end points $P_{0}$ and $P$ and the constant of integration $\sigma_{0}\left(\varphi_{0}, \vartheta_{0}\right)$ can be obtained from the stress boundary conditions $(2.2)$ and (2.3).

\section{EXAMPLeS}

4.1 Example 1. Lamé's problem for a hollow circular cone. The body considered in this example is bounded by two spherical surfaces $\partial V_{i}(i=1,2)$ of radius $r_{i}$ $(i=1,2)$ and two circular conical surfaces $\partial V_{i}(i=3,4)$ whose mantles are given by the following prescription

$$
\vartheta=\vartheta_{i} \quad \text { on } \quad \partial V_{i}(i=3,4) .
$$

The meridian section of this body of rotation is shown in Figure 3 .

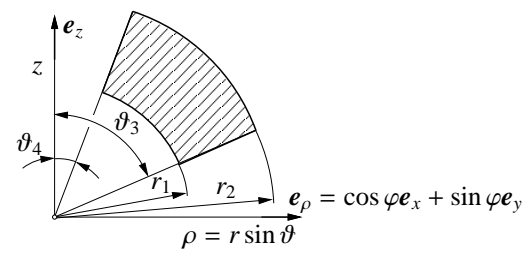

Figure 3. Meridian section of body of rotation

The surface forces on $\partial V_{3}$ and $\partial V_{4}$ are given by

$$
\widetilde{\boldsymbol{p}}_{3}=-p_{3} \boldsymbol{e}_{\vartheta}, \quad \widetilde{\boldsymbol{p}}_{4}=p_{4} \boldsymbol{e}_{\vartheta} \quad p_{3} \text { and } p_{4} \text { are constants, } \quad\left(p_{3}>0, p_{4}>0\right) .
$$

It is easy to show that the solvability condition 2.15 for this boundary value problem is fulfilled since

$$
-\int_{\partial a_{3}} p_{3} \boldsymbol{e}_{r} \times \boldsymbol{e}_{\vartheta} \mathrm{d} s_{3}+\int_{\partial a_{4}} p_{4} \boldsymbol{e}_{r} \times \boldsymbol{e}_{\vartheta} \mathrm{d} s_{4}=-p_{3} \sin \vartheta_{3} \int_{0}^{2 \pi} \boldsymbol{e}_{\varphi} \mathrm{d} \varphi+p_{4} \sin \vartheta_{4} \int_{0}^{2 \pi} \boldsymbol{e}_{\varphi} \mathrm{d} \varphi=\mathbf{0} .
$$

If take into account that Example 4.1 is an axially symmetric statical boundary value problem we can write

$$
\begin{array}{r}
U_{\vartheta}=U_{\vartheta}(\vartheta), \quad U_{\varphi}=0, \quad \varepsilon_{\vartheta}=\varepsilon_{\vartheta}(\vartheta), \quad \varepsilon_{\varphi}=\varepsilon_{\varphi}(\vartheta), \quad \gamma_{\varphi \vartheta}=0, \\
\sigma_{\vartheta}=\sigma_{\vartheta}(\vartheta), \quad \sigma_{\varphi}=\sigma_{\varphi}(\vartheta), \quad \sigma_{0}=\sigma_{0}(\vartheta), \quad \tau_{\varphi \vartheta}=0 .
\end{array}
$$

Solutions for the displacement potential are sought in the form

$$
F=C \varphi,
$$


where $C$ is an arbitrary constant. By a routine computation it easy to see that the displacement potential given by equation (4.5) satisfies equations 3.22 and $(3.23)$. In addition it follows from equations (3.17) and (3.16) that

$$
\frac{\partial \sigma_{0}}{\partial \varphi}=0, \quad \frac{\partial \sigma_{0}}{\partial \vartheta}=-2 G \frac{C}{\sin \vartheta} .
$$

Integration of equation 4.6 yields

$$
\sigma_{0}(\vartheta)=-2 G C \ln \left|\tan \frac{\vartheta}{2}\right|+D
$$

where $D$ is a constant of integration. Making use of equations (3.6), (3.7), (3.8) and (4.7) we have

$$
\begin{gathered}
\sigma_{\vartheta}=-2 G C\left(\frac{\cos \vartheta}{\sin ^{2} \vartheta}+\ln \left|\tan \frac{\vartheta}{2}\right|\right)+D, \\
\sigma_{\varphi}=2 G C\left(\frac{\cos \vartheta}{\sin ^{2} \vartheta}-\ln \left|\tan \frac{\vartheta}{2}\right|\right)+D, \\
\sigma_{r}=-2 G C \ln \left|\tan \frac{\vartheta}{2}\right|+D .
\end{gathered}
$$

The unknown integration constants $C$ and $D$ can now be calculated from the stress boundary conditions

$$
\sigma_{\vartheta}\left(\vartheta_{3}\right)=-p_{3}, \quad \sigma_{\vartheta}\left(\vartheta_{4}\right)=-p_{4}
$$

Omitting the details we get

$$
\begin{gathered}
C=\frac{p_{3}-p_{4}}{2 G\left[\ln \left|\frac{\tan \frac{\vartheta_{3}}{2}}{\tan \frac{\vartheta_{4}}{2}}\right|+\frac{\cos \vartheta_{3}}{\sin ^{2} \vartheta_{3}}-\frac{\cos \vartheta_{4}}{\sin ^{2} \vartheta_{4}}\right]}, \\
D=\frac{p_{3}\left(\frac{\cos \vartheta_{4}}{\sin ^{2} \vartheta_{4}}-\ln \left|\tan \frac{\vartheta_{4}}{2}\right|\right)-p_{4}\left(\frac{\cos \vartheta_{3}}{\sin ^{2} \vartheta_{3}}-\ln \left|\tan \frac{\vartheta_{3}}{2}\right|\right)}{\ln \left|\frac{\tan \frac{\vartheta_{3}}{2}}{\tan \frac{\vartheta_{4}}{2}}\right|+\frac{\cos \vartheta_{3}}{\sin ^{2} \vartheta_{3}}-\frac{\cos \vartheta_{4}}{\sin ^{2} \vartheta_{4}}} .
\end{gathered}
$$

Once we know $C$ equation 4.5 yields $F$. Then the non-zero displacement component $u_{\vartheta}$ can be obtained from 3.1 :

$$
u_{\vartheta}=u_{\vartheta}(r, \vartheta)=\frac{\left(p_{3}-p_{4}\right) r}{2 G\left[\ln \left|\frac{\tan \frac{\vartheta_{3}}{2}}{\tan \frac{\vartheta_{4}}{2}}\right|+\frac{\cos \vartheta_{3}}{\sin ^{2} \vartheta_{3}}-\frac{\cos \vartheta_{4}}{\sin ^{2} \vartheta_{4}}\right] \sin \vartheta} .
$$

For $p_{3}=p_{4}=p$, we have

$$
\begin{gathered}
C=0, \quad D=-p, \\
\sigma_{r}=\sigma_{\varphi}=\sigma_{\vartheta}=\sigma_{0}=-p, \quad u_{\vartheta}=0
\end{gathered}
$$

which represent a hydrostatic state of stresses.

The formulas derived above are used in the following numerical example:

$$
\begin{aligned}
\vartheta_{3}=\frac{\pi}{3}, \quad \vartheta_{4}=\frac{\pi}{6}, \quad G=0.1 \times 10^{9} \mathrm{~Pa} & \text { (rubber) } \\
& p_{3}=1 \times 10^{6} \mathrm{~N} / \mathrm{m}^{2}, \quad p_{4}=4 \times 10^{6} \mathrm{~N} / \mathrm{m}^{2} .
\end{aligned}
$$




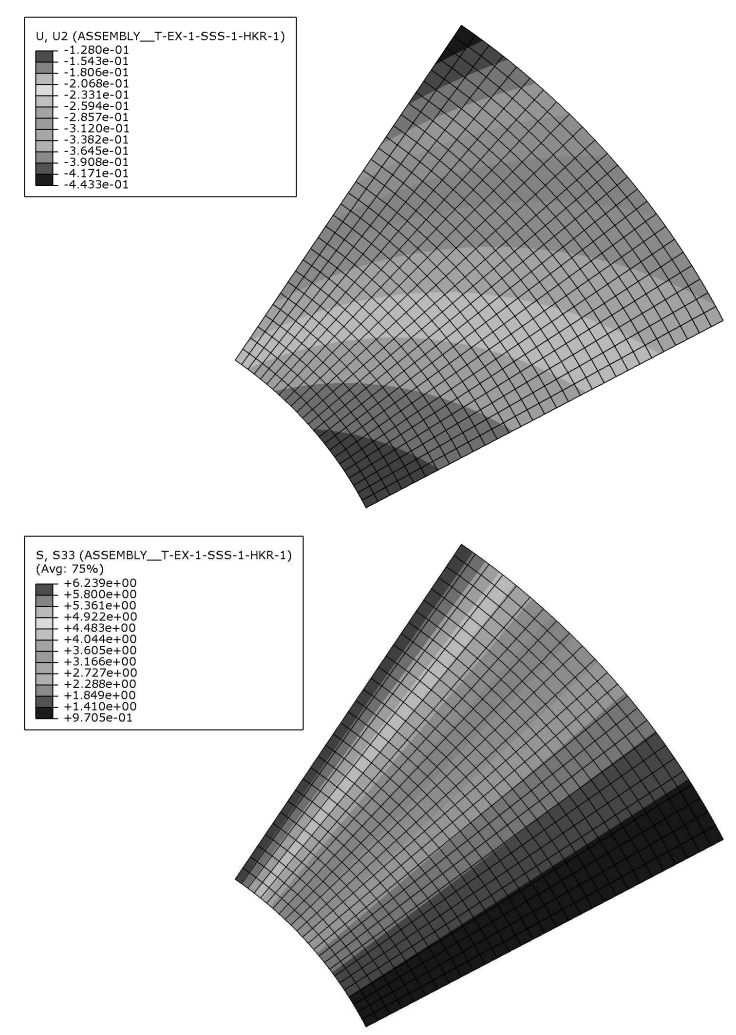

Figure 4. $\mathrm{U} 2=u_{\vartheta}$ and $\mathrm{S} 33=\sigma_{\varphi}$ from FEM computations

The stress values are independent of the magintude of $r_{i}(i=1,2)$. Figure 5 shows the graph of $\sigma_{0}=\sigma_{0}(\vartheta), \sigma_{\vartheta}=\sigma_{\vartheta}(\vartheta), \sigma_{\varphi}=\sigma_{\varphi}(\vartheta)$. The graph of $u_{\vartheta}(1, \vartheta)=U_{\vartheta}(\vartheta)$ is shown in Figure 6 .

According to equation 2.10

$$
\begin{gathered}
2 \int_{a} \sigma_{r} \boldsymbol{e}_{r} \mathrm{~d} a=1.570796327 \times 10^{6} \boldsymbol{e}_{z} \mathrm{~N} / \mathrm{m}^{2} \\
\int_{\partial a_{3}} \widetilde{\boldsymbol{p}}_{3} \mathrm{~d} s_{3}+\int_{\partial a_{4}} \widetilde{\boldsymbol{p}}_{4} \mathrm{~d} s_{4}=-1.570796327 \times 10^{6} \boldsymbol{e}_{z} \mathrm{~N} / \mathrm{m}^{2} .
\end{gathered}
$$

The FEM solutions shown in Figures 4, 5 and 6 are computed by the finite element software Abaqus using $\mathrm{CAX} 8 \mathrm{H}$, which are 8-node biquadratic hybrid elements with linear pressure approximation. 


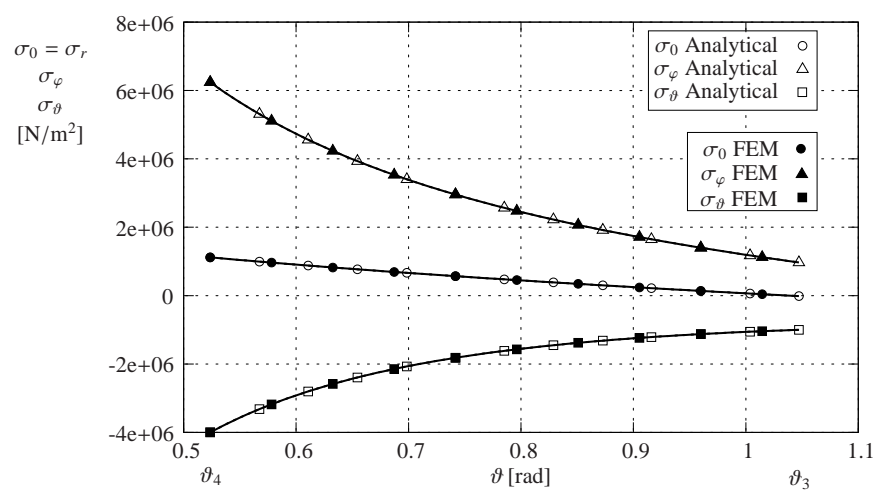

Figure 5. Graphs of normal stresses. Analytical and FEM solutions

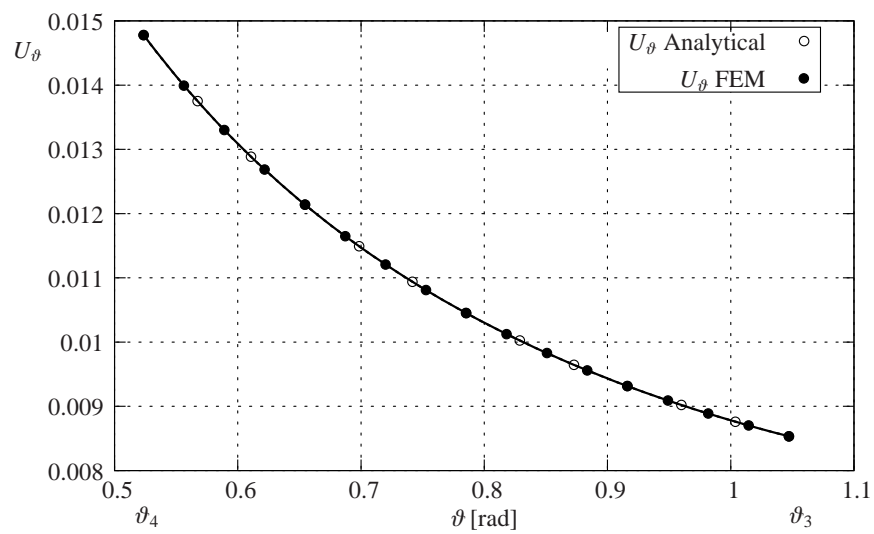

Figure 6. Graph of $u_{\vartheta}(1, \vartheta)=U_{\vartheta}(\vartheta)$

4.2 Example 2. Torsional spring. For the problem examined in this section the displacement boundary conditions on the boundary surface segment $\partial V_{3} \cup \partial V_{4}$ are of the form

$$
\begin{gathered}
\boldsymbol{u}=\widetilde{\boldsymbol{u}}_{3}=\mathbf{0} \quad \text { on } \quad \partial V_{3}=\left\{(r \varphi, \vartheta) \mid r_{1} \leq r \leq r_{2}, 0 \leq \varphi \leq 2 \pi, \vartheta=\vartheta_{3}\right\}, \\
\boldsymbol{u}=\widetilde{\boldsymbol{u}}_{4}=r \alpha \sin \vartheta_{4} \boldsymbol{e}_{\varphi} \quad \text { on } \quad \partial V_{3}=\left\{(r, \varphi, \vartheta) \mid r_{1} \leq r \leq r_{2}, 0 \leq \varphi \leq 2 \pi, \vartheta=\vartheta_{4}\right\} .
\end{gathered}
$$

It is clear that

$$
\begin{gathered}
U_{\vartheta}=0 \text { on } \partial V_{3} \cup \partial V_{4}, \\
U_{\varphi}=0 \quad \text { on } \partial V_{3} \text { and } U_{\varphi}=\alpha \sin \vartheta \quad \text { on } \partial V_{4} .
\end{gathered}
$$

Note that the solvability condition 2.17 is satisfied by the surface displacements (4.22. 


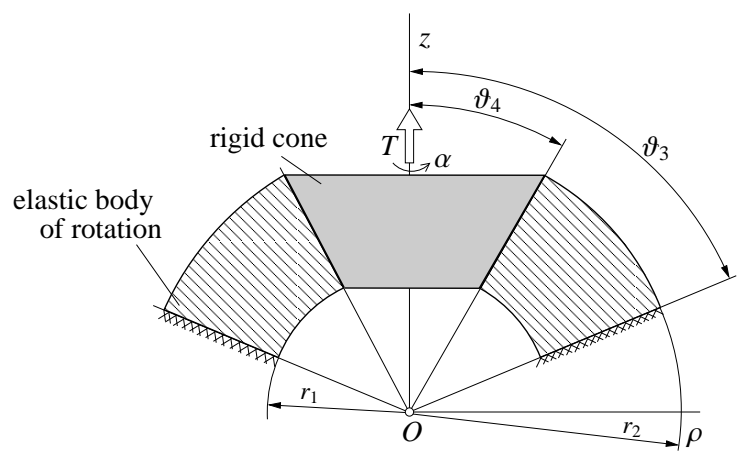

Figure 7. Example for prescribed surface displacements

Figure 7 shows the meridian section of the coupled system of an elastic-rigid body with rotational symmetry. The rigid cone is rotated by angle $\alpha$ about the axis $z$. This rotation creates that surface displacement on the boundary surface $\partial V_{4}$ which is given by equation 4.20 . The rotation of the rigid cone about the axis $z$ is produced by a torque $T$ exerted on the cone as shown in Figure 7 . Solution to the boundary value problem defined above is derived from the displacement potential

$$
F(\vartheta, \varphi)=\frac{C_{1}}{2} \cos \vartheta \ln \left|\tan \frac{\vartheta}{2}\right|+C_{2} \cos \vartheta
$$

It is very easy to show that in the present problem

$$
L_{\vartheta}[F]=L_{\varphi}[F]=0 .
$$

It follows from equation 3.1 that

$$
\begin{gathered}
U_{\vartheta}=0 \\
U_{\varphi}=\frac{C_{1}}{2}\left(\sin \vartheta \ln \left|\tan \frac{\vartheta}{2}\right|-\cot \vartheta\right)+C_{2} \sin \vartheta .
\end{gathered}
$$

With $U_{\varphi}$ equations 1.2 and 1.3 yield

$$
\varepsilon_{\vartheta}=\varepsilon_{\varphi}=0, \quad \gamma_{\varphi \vartheta}=\frac{C_{1}}{\sin ^{2} \vartheta} .
$$

A combination of equations (1.4), 1.5), 1.6), 1.9 and 4.2 with equations 4.27) results in the stresses:

$$
\sigma_{r}=\sigma_{\vartheta}=\sigma_{\varphi}=\sigma_{0}=D=\text { constant. }
$$

The equilibrium condition for the rigid circular cone leads to the equation

$$
\sigma_{\vartheta}=D=0 .
$$

Figure 8 is the free-body diagram of the rigid cone. Note that equation 4.29 is a consequence of the force equilibrium in the direction of the axis $z$. 


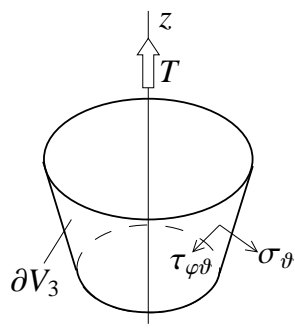

Figure 8. Free-body diagram of the rigid cone

The integration constants $C_{1}$ and $C_{2}$ are obtained from the displacement boundary conditions $4.221_{1,2}$. A detailed computation yields

$$
C_{1}=\frac{H_{1}}{H}, \quad C_{2}=\frac{H_{2}}{H},
$$

where

$$
\begin{gathered}
H=2 \sin \vartheta_{3}\left(\sin \vartheta_{4} \ln \left|\tan \frac{\vartheta_{4}}{2}\right|-\cot \vartheta_{4}\right)-2 \sin \vartheta_{4}\left(\sin \vartheta_{3} \ln \left|\tan \frac{\vartheta_{3}}{2}\right|-\cot \vartheta_{3}\right), \\
H_{1}=4 \alpha \sin \vartheta_{3} \sin \vartheta_{4} \\
H_{2}=-2 \alpha \sin \vartheta_{4}\left(\sin \vartheta_{3} \ln \left|\tan \frac{\vartheta_{3}}{2}\right|-\cot \vartheta_{3}\right) .
\end{gathered}
$$

Making use of equations $(3.9)$ and $(4.27), 4.301,4$, 4.31) then equation (4.32), we get the shearing stress:

$$
\tau_{\varphi \vartheta}=\frac{2 \alpha G}{\left(h\left(\vartheta_{4}\right)-h\left(\vartheta_{3}\right)\right) \sin ^{2} \vartheta}
$$

where

$$
h(\vartheta)=\ln \left|\tan \frac{\vartheta}{2}\right|-\frac{\cos \vartheta}{\sin ^{2} \vartheta}
$$

Relationship between the applied torque and the prescribed angular displacement $\alpha$ can be derived from the moment equilibrium equation:

$$
T+2 \pi \int_{r_{1}}^{r_{2}} \tau_{\varphi \vartheta} r^{2} \sin \vartheta \mathrm{d} r=0 .
$$

Omitting the details we get

$$
T=R \alpha, \quad R=\frac{4}{3} \pi G \frac{r_{2}^{3}-r_{1}^{3}}{h\left(\vartheta_{3}\right)-h\left(\vartheta_{4}\right)} .
$$

Figures 10 and 11 show the graph of functions $\tau_{\vartheta \varphi}=\tau_{\vartheta \varphi}(\vartheta)$ and $u_{\varphi}(1, \vartheta)=U_{\varphi}(\vartheta)$. The following data were used $\vartheta_{3}=\frac{\pi}{2}, \vartheta_{4}=\frac{\pi}{6}, G=10^{8} \mathrm{~N} / \mathrm{m}^{2}, \alpha=10^{-2} \mathrm{rad}$, $r_{1}=0.01 \mathrm{~m}, r_{2}=0.02 \mathrm{~m}$. In this example

$$
R=613.28522 \mathrm{Nm}, \quad T=6.1328522 \mathrm{Nm} .
$$



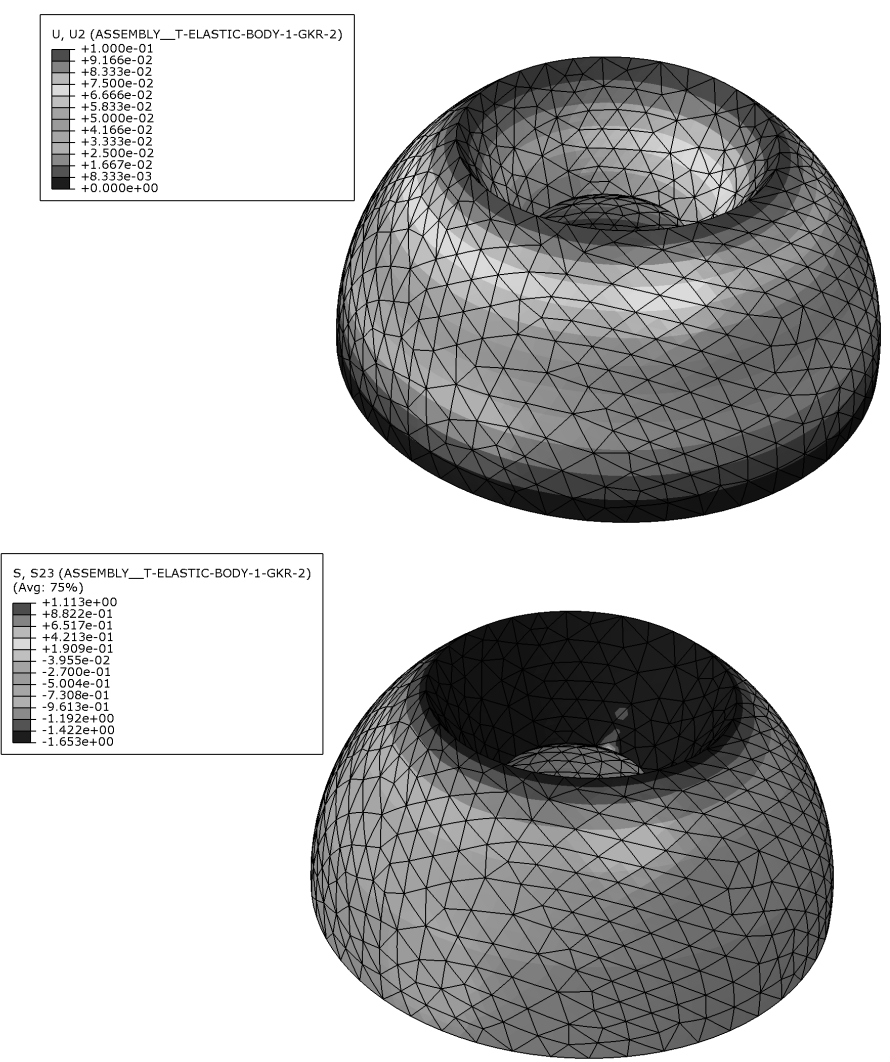

Figure 9. U2 $=u_{\varphi}$ and $\mathrm{S} 23=\tau_{\varphi \vartheta}$ from FEM computations

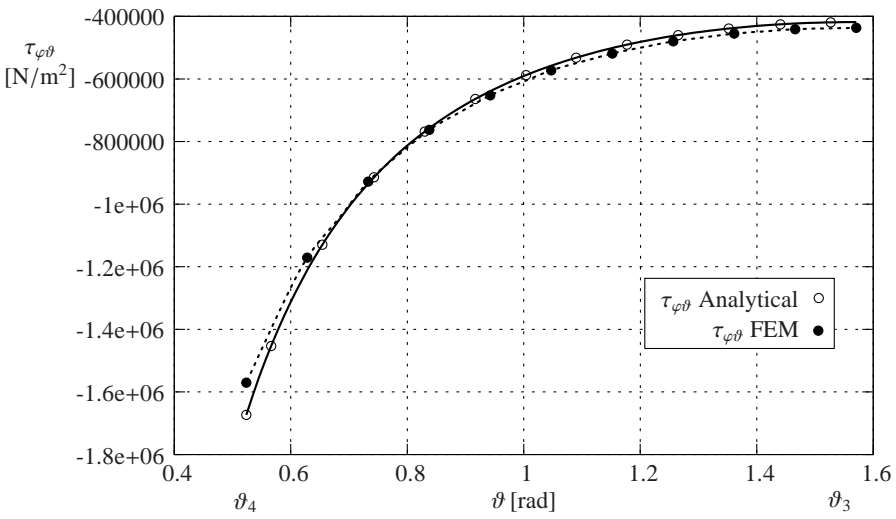

Figure 10. Analytical and FEM solutions for the shearing stresses 


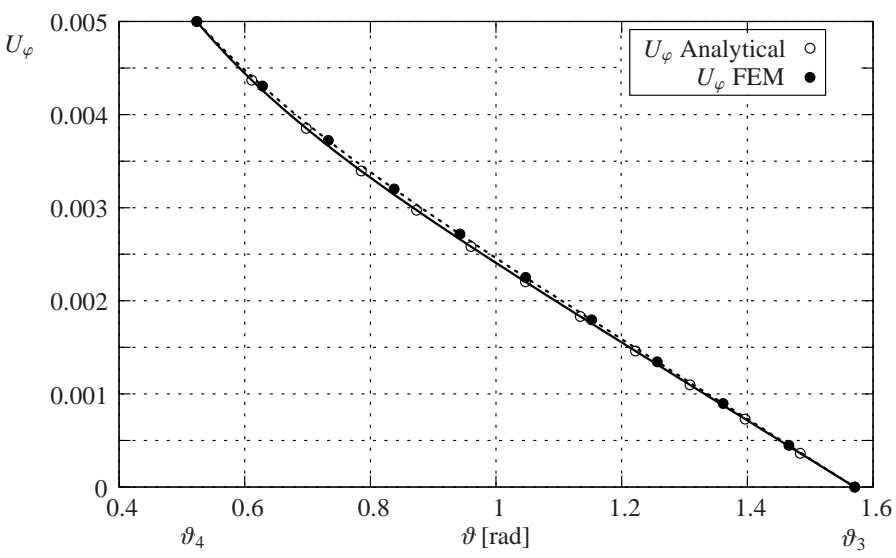

Figure 11. Analytical and FEM solutions for $u_{\varphi}(1, \vartheta)=U_{\varphi}(\vartheta)$

The results of FEM computation are represented in Figure 9 Figures 10 and 11 show the comparison of the analytical and FEM solutions. The latter are computed by the finite element software Abaqus using the 10-node quadratic tetrahedron hybrid finite element $\mathrm{C} 3 \mathrm{D} 10 \mathrm{H}$ with constant pressure approximation.

\section{Conclusions}

In this paper the static equilibrium of an elastic body made of incompressible and homogeneous material is investigated. We have assumed that the body considered is bounded by two spherical and two conical surfaces. We have introduced a new concept called spherical strain state (SSS). If a body is in SSS the strains and stresses are independent of the radial coordinate in a properly chosen spherical coordinate system.

The present paper is a contribution to the existing exact benchmark solutions for incompressible elastic bodies. The analytical results we have obtained for the two examples are compared with finite element solutions. They are in excellent agreement.

The Poisson number for the finite element solutions, which are computed by the software Abaqus, is $\nu=0.499999$ for both examples.

\section{ACKNOWLEDGMENT}

This research was (partially) carried out in the framework of the Center of Excellence of Innovative Engineering Design and Technologies at the University of Miskolc and supported by the National Research, Development and Innovation Office - NKFIH, K115701. 


\section{REFERENCES}

1. BabuskA, I. and SuRI, M.: Locking Effects in the Finite Element Approximation of Elasticity Problems. Technical Note BN-1119. Institute for Physical Science and Technology University of Maryland, 1990.

2. Szabó, B. and Babuška, I.: Finite Element Analysis. John Wiley, New York, 1991.

3. Gurtin, M. E.: The Linear Theory of Elasticity in Handbuch der Physik. VI/2. (Ed. Flüge. S.) Springer, Berlin, 1972.

4. Slaughter, W. E.: The Linearized Theory of Elasticity. Birkhäuser, Boston, 2001.

5. Sokolnikoff, I. S.: Mathematical Theory of Elasticity. (2nd ed.) McGraw-Hill, New York, 1956. 